\title{
SUPERDIAGONAL FORMS FOR RELATED LINEAR OPERATORS
}

\author{
by VOLKER WROBEL
}

(Received 17 August, 1983)

The concept of superdiagonal forms for $n \times n$ matrices $T$ with complex entries has been extended by J. R. Ringrose [4] to the setting of compact linear operators $T: X \rightarrow X$ acting on a complex Banach space $X$. In a recent paper $D$. Koros [2] generalized Ringrose's approach to the case of compact linear operators $T: X \rightarrow X$ on a complex locally convex space $X$. The reason why both authors confine their attention to the class of compact linear operators is that the existence of proper closed invariant subspaces is, aside from Riesz-Schauder theory, the main tool in their construction. In the present paper it is shown that the existence of superdiagonal forms possesses a certain permanence property in the following sense.

Let $X$ and $Y$ denote two locally convex spaces, let $P: X \rightarrow Y$ and $Q: Y \rightarrow X$ denote two continuous linear operators. Then according to $A$. Pietsch [3] the operators $T:=Q P: X \rightarrow X$ and $S:=P Q: Y \rightarrow Y$ are said to be related. Roughly speaking we shall prove that $T$ has a superdiagonal form in the sense of Ringrose if and only if $S$ has a superdiagonal form. Since every compact linear operator $T: X \rightarrow X$ on a complex locally convex space $X$ is related to a compact linear operator $S: Y \rightarrow Y$ on a complex Banach space $Y$, we especially obtain an independent approach to Koros's result without using any locally convex arguments concerning Riesz-Schauder theory and invariant subspaces.

1. Notation. Throughout this paper $X$ and $Y$ denote locally convex spaces over the complex numbers, $L(X, Y)$ denotes the space of all continuous linear operators from $X$ to $Y$, writing $L(X)$ for $L(X, X)$. The term subspace will always mean closed linear subspace, and a proper subspace will be a subspace different from $\{0\}$ and the whole space. A subspace $M$ of $X$ is said to be invariant under $T: X \rightarrow X$ if $T x \in M$ for all $x \in M$. A nest $\mathscr{F}$ of subspaces of $X$ will be a family of subspaces which is totally ordered by inclusion. If in addition every $M \in \mathscr{F}$ is invariant under $T, \mathscr{F}$ will be said to be an invariant nest. The symbol " $\subset$ " will be reserved for proper inclusion. If $S \subset X$ is a subset, let cl $S$ denote the closure of $S$ with respect to the topology of $X$.

Given a nest $\mathscr{F}$ of subspaces of $X$ containing $\{0\}$ and $X$, for $M \in \mathscr{F}$ define

$$
\begin{aligned}
& M_{-}=\operatorname{cl} \bigcup\{L: L \in \mathscr{F}, L \subset M\}, \\
& M_{+}=\bigcap\{L: L \in \mathscr{F}, L \supset M\} .
\end{aligned}
$$

Obviously $M_{-}$and $M_{+}$are subspaces of $X$, and it may happen that $M_{-}=\{0\}$ while $M_{+}=X$. $M_{-}$and $M_{+}$are invariant under $T$ provided $\mathscr{F}$ is an invariant nest.

DefintTION 1.1. A nest $\mathscr{F}$ of subspaces of $X$ is said to be simple if

(i) $\{0\}, X \in \mathscr{F}$;

Glasgow Math. J. 26 (1985) 19-23. 
(ii) if $\mathscr{F}_{0}$ is any subfamily of $\mathscr{F}$, then the subspaces $\bigcap\left\{L: L \in \mathscr{F}_{0}\right\}$ and cl $\bigcup\left\{L: L \in \mathscr{F}_{0}\right\}$ are in $\mathscr{F}$;

(iii) if $M \in \mathscr{F}$, then $\operatorname{dim} M / M_{-} \leqslant 1$.

Condition (iii) can be replaced by the equivalent condition

(iii) ${ }^{\prime}$ if $M \in \mathscr{F}$, then $\operatorname{dim} M_{+} / M \leqslant 1$.

In order to see this, assume $M \subset M_{+}$. Then $M \subseteq M_{+-}$. On the other hand, if $L \in \mathscr{F}$ is such that $L \subset M_{+}$, then $L \subseteq M$, for otherwise $L \supseteq M_{+}$. Hence $M_{+-}=M$ and (iii) implies (iii)'. Conversely assume that (iii)' holds. Thus let $M_{-} \subset M$. By definition we have $M_{-_{+}} \subseteq M$. On the other hand, if $L \in \mathscr{F}$ and $L \supset M_{-}$, then $L \supseteq M$, for otherwise $L \subset M$ and hence $L \subset M_{-}$. This gives $M_{-+}=M$.

A linear operator $T: X \rightarrow X$ is said to be compact if there exists a neighbourhood $U$ of zero in $X$ such that $T(U)$ is relatively compact. Letting $Y$ denote the linear span of cl $T(U)$ provided with the Minkowski-norm

$$
\dot{m_{Y}}(y):=\inf \left\{c>0: c^{-1} y \in c l T(U)\right\},
$$

it is easily seen that $T$ factors compactly through $Y$. Indeed $T=Q P$, where $P: X \rightarrow Y$ is given by $P x=T x$ for $x \in X$, and $Q$ denotes the embedding of $Y$ into $X$, this map also being compact. Therefore $T$ is related to the compact operator $S$ defined to be $P Q$ acting on the Banach space $Y$.

2. Simple invariant nests for related linear operators. Given an arbitrary operator $T \in L(X)$ and an invariant nest $\mathscr{F}$ we can always find a maximal invariant nest $\mathscr{F}_{\max }$ containing $\mathscr{F}$ by an argument based on Zorn's lemma, but in general a maximal invariant nest will not be simple. Indeed by Enflo's counterexample to the invariant subspace problem [1] there exists a Banach space $X$ and $T \in L(X)$ such that $\{0\}, X$ is a maximal invariant nest for $T$. Since superdiagonal forms are given for simple nest only, we confine our attention to those operators for which a maximal invariant nest is automatically simple and related operators.

Theorem 2.1. Let $T \in L(X)$ and $S \in L(Y)$ denote two related linear operators. If every maximal invariant nest for $T$ is simple, then the same is true for $S$.

Proof. Let $\mathscr{F}$ be a maximal invariant nest for $S$, let $M \in \mathscr{F}$ and assume that $\operatorname{dim} M / M_{-}>1$. Obviously $P^{-1}(M)$ and $P^{-1}\left(M_{-}\right)$are invariant subspaces for $T$, and since $Q M \subseteq P^{-1}(M)(M$ is invariant under $S)$, the restriction of $T$ to $P^{-1}(M)$ is related to the restriction of $S$ to $M$. We shall prove that $\operatorname{dim} M / M_{-}>1$ is impossible. For that purpose we may assume that $X=P^{-1}(M)$ and $Y=M$. On the other hand $\hat{T}: X / P^{-1}\left(Y_{-}\right) \rightarrow$ $X / P^{-1}\left(Y_{-}\right)$given by $\hat{T}([x])=[T x]$ and $\hat{S}: Y / Y_{-} \rightarrow Y / Y_{-}$given by $\hat{S}(\langle y\rangle)=\langle S y\rangle$ are related, [.] and $\langle$.$\rangle denoting the cosets in X / P^{-1}\left(Y_{-}\right)$and $Y / Y_{-}$respectively. Indeed $\hat{T}=\hat{Q} \hat{P}$, $\hat{S}=\hat{P} \hat{Q}$ with $\hat{P}([x])=\langle P x\rangle$ and $\hat{Q}(\langle y\rangle)=[Q y]$. Thus, if $\operatorname{dim} X / P^{-1}\left(Y_{-}\right) \leqslant 1$, then $\hat{S}$ has at most rank one. Consequently, we find a proper invariant subspace $\hat{N}$ of $\hat{S}$ and $N:=\{y:\langle y\rangle \in \hat{N}\}$ is an invariant subspace of $S$ such that $Y_{-} \subset N \subset Y$. It is easily checked that $\{N\} \cup \mathscr{F}$ is an invariant nest containing $\mathscr{F}$ properly, contradicting the maximality of $\mathscr{F}$. Therefore let us assume that $\operatorname{dim} X / P^{-1}\left(Y_{-}\right)>1$. Since $X$ and $P^{-1}\left(Y_{-}\right)$are members of a 
suitable simple invariant nest $\mathscr{G}$, there exists $N \in \mathscr{G}$ such that $P^{-1}\left(Y_{-}\right) \subset N \subset X$. Then $Q^{-1}(N)$ is an invariant subspace for $S$. We shall show that $N$ can be chosen in such a way that $Y_{-} \subset Q^{-1}(N) \subset Y$. This again will contradict the maximality of $\mathscr{F}$ and hence finish the proof. First of all we remark that for $L \in \mathscr{G}, Q^{-1}(L)=Y_{-}$implies $L \subseteq T^{-1}(L)=P^{-1}\left(Y_{-}\right)$. Consider

$$
P^{-1}\left(Y_{-}\right)_{+}=\bigcap\left\{K: K \in \mathscr{G}, K \supset P^{-1}\left(Y_{-}\right)\right\}
$$

If we had $Q^{-1}(K)=Y$ for all such $K$, then $Q^{-1}\left(P^{-1}\left(Y_{-}\right)_{+}\right)=Y$ (otherwise we are done!). We distinguish two cases.

(i) If $P^{-1}\left(Y_{-}\right)_{+}=P^{-1}\left(Y_{-}\right)$, then $S^{-1}\left(Y_{-}\right)=Q^{-1}\left(P^{-1}\left(Y_{-}\right)_{+}\right)=Y$. Thus $\hat{S}: Y / Y_{-} \rightarrow$ $Y / Y_{-}$is identically zero.

(ii) If $P^{-1}\left(Y_{-}\right)_{+} \neq P^{-1}\left(Y_{-}\right)$, then $P^{-1}\left(Y_{-}\right)_{+}=P^{-1}\left(Y_{-}\right) \oplus \mathbb{C} x_{0}$ with a suitable $x_{0} \in X$, because $\mathscr{G}$ was a simple nest. Therefore

$$
S Y=P Q\left(Q^{-1}\left(P^{-1}\left(Y_{-}\right) \oplus C x_{0}\right)\right) \subseteq Y_{-}+\mathbb{C} P x_{0},
$$

and hence $\hat{S}: Y / Y_{-} \rightarrow Y / Y_{-}$is at most of rank one. In both cases $\hat{S}$ has a proper invariant subspace. By the argument used at the beginning of the proof we obtain a contradiction to F being maximal. Thus $\operatorname{dim} M / M_{-}$is at most one and $\mathscr{G}$ is simple.

Throughout the remainder of this section, let $T=Q P \in L(X)$ and $S=P Q \in L(Y)$ denote two related linear operators such that every maximal invariant nest of subspaces is simple. Let $\mathscr{F}(T)$ denote a simple invariant nest for $T$; then $\mathscr{F}(S)$ and $\mathscr{F}_{-1}(T)$ denote simple invariant nests for $S$ and $T$ containing $\left\{Q^{-1}(M): M \in \mathscr{F}(T)\right\}$ and $\left\{P^{-1}(K): K \in \mathscr{F}(S)\right\}$, respectively.

If $M \in \mathscr{F}(T)$, we have $M=M_{-}$or $\operatorname{dim} M / M_{-}=1$. Let us assume $M \neq M_{-}, z_{M} \in$ $M \backslash M_{-}$. Then $T z_{M} \in M$ can be expressed uniquely in the form

$$
T z_{M}=\alpha_{M}(T) z_{M}+y_{M}
$$

where $\alpha_{M}(T) \in \mathbb{C}$ and $y_{M} \in M_{-}$. The scalar $\alpha_{M}(T)$ does not depend on the choice of $z_{M}$. In this way we can associate to each $M \in \mathscr{F}(T)$ a complex number $\alpha_{M}(T)$ called the diagonal coefficient of $T$ at $M$. Let $\alpha$ be a scalar. We define the diagonal multiplicity of $\alpha$ to be the (possibly infinite) number of distinct subspaces $M \in \mathscr{F}(T)$ for which $\alpha_{M}(T)=\alpha$.

TheOREM 2.2. Let $T \in L(X)$ and $S \in L(Y)$ denote two related linear operators. Then there is a one-to-one correspondence between the diagonal coefficient of $T$ with respect to $\mathscr{F}(T)$ and those of $S$ with respect to $\mathscr{F}(S)$. More precisely: given $\alpha \in \mathbb{C} \backslash\{0\}$, the diagonal multiplicity of $\alpha$ is the same with respect to both $\mathscr{F}(T)$ and $\mathscr{F}(S)$.

Proof. Let $M \in \mathscr{F}(T)$ and assume that $\alpha_{M}(T) \neq 0$. Then $M=M_{-} \oplus \mathbb{C} z_{M}$ with a suitable $z_{M} \in M \backslash M_{-}$. Then $T z_{M} \notin M_{-}$, and hence $P z_{M} \notin Q^{-1}\left(M_{-}\right)$. On the other hand $M=M_{-} \oplus \mathbb{C} T z_{M}$, and therefore

$$
Q^{-1}(M)=Q^{-1}\left(M \oplus \mathbb{C} Q P z_{M}\right)=Q^{-1}\left(M_{-}\right) \oplus \mathbb{C} P z_{M}
$$

This implies $Q^{-1}(M)_{-}=Q^{-1}\left(M_{-}\right)$. Since $S P z_{M}=P T z_{M}=P\left(\alpha_{M}(T) z_{M}+y_{M}\right)=$ $\alpha_{M}(T) P z_{M}+P y_{M}\left(P y_{M} \in Q^{-1}\left(M_{-}\right)\right)$, we have $\alpha_{M}(T)=\alpha_{Q^{-1}(M)}(S)$. If $M_{1} \subset M_{2}\left(M_{i} \in \mathscr{F}(T)\right)$, 
then $\alpha_{M_{i}}(T) \neq 0$ implies $Q^{-1}\left(M_{1}\right) \subset Q^{-1}\left(M_{2}\right)$, for otherwise $T M_{2} \subseteq M_{1}$, which gives $\alpha_{M_{2}}(T)=0$, a contradiction. Therefore the diagonal multiplicity of $\alpha \neq 0$ with respect to $\mathscr{F}(S)$ exceeds that of $\alpha$ with respect to $\mathscr{F}(T)$.

Conversely let $K \in \mathscr{F}(S)$ and $\alpha_{K}(S) \neq 0$. Define

$$
M_{-}=\bigcap\left\{M: M \in \mathscr{F}(T), Q^{-1}(M) \supset K\right\} .
$$

Of course $M_{\sim} \in \mathscr{F}(T)$. On the other hand we prove that $L=M_{\sim} \cap P^{-1}(K) \in \mathscr{F}(T)$. So let $N \in \mathscr{F}(T)$. If $Q^{-1}(N) \subset K$, then $Q^{-1}(N) \subseteq Q^{-1}\left(M_{\sim}\right)$, and $N \subseteq M_{\sim}, T^{-1}(N) \subseteq P^{-1}(K)$. This gives $N \subseteq M_{\sim} \cap P^{-1}(K)$. If $Q^{-1}(N) \supseteq K$, then $Q^{-1}(N) \supseteq Q^{-1}\left(M_{\sim}\right)$ and $N \supseteq M_{\sim}$, which implies $N \supseteq M_{\sim} \cap P^{-1}(K)$. By the maximality of $\mathscr{F}(T), \quad L \in \mathscr{F}(T)$. Moreover $M_{\sim} \cap P^{-1}(K)_{-}$has at most codimension one in $L$. Note that $P^{-1}(K)_{-}$(with respect to $\mathscr{F}_{-1}(T)$ of course!) equals $P^{-1}\left(K_{-}\right)$by the same argument as in the first step of this proof. If we had $L=M_{\sim} \cap P^{-1}\left(K_{-}\right)$, then $Q^{-1}(L)=S^{-1}\left(K_{-}\right) \cap Q^{-1}\left(M_{\sim}\right)$, and hence $K \cap S^{-1}(K) \cap Q^{-1}\left(M_{\sim}\right)=K \cap S^{-1}\left(K_{-}\right) \cap Q^{-1}\left(M_{\sim}\right)$; i.e. $K=K_{-}$, contradicting $\alpha_{K}(S) \neq 0$. Thus $L_{-}=P^{-1}\left(K_{-}\right) \cap M_{\sim}$. If we had $T L \subseteq L_{-}$, then $S^{2}(K)=S^{2}\left(K \cap Q^{-1}\left(M_{\sim}\right)\right) \subseteq$ $S\left(S K \cap S\left(Q^{-1}\left(M_{\sim}\right)\right) \subseteq S\left(K \cap P Q\left(Q^{-1}\left(M_{\sim}\right)\right) \subseteq S\left(K \cap P M_{\sim}\right)=S\left(P\left(P^{-1}(K) \cap P M_{\sim}\right)=\right.\right.\right.$ $P T\left(P^{-1}(K) \cap M_{\sim}\right)=P T(L) \subseteq P L_{-}=P\left(P^{-1}\left(K_{-}\right) \cap M_{\sim}\right) \subseteq K_{-}$; i.e. $S^{2}$ maps $K$ into $K_{-}$contradicting $\alpha_{K}(S) \neq 0$. Thus $\alpha_{L}(T) \neq 0$. Let $y_{0} \in K \backslash K_{-}$. Then $Q y_{0} \in P^{-1}(K) \backslash P^{-1}\left(K_{-}\right)$as in the first step and $L=L_{-} \oplus \mathbb{C} Q y_{0}$. Hence $T Q y_{0}=Q S y_{0}=Q\left(\alpha_{K}(S) y_{0}+y_{M}\right)=$ $\alpha_{K}(S) Q y_{0}+Q y_{k}\left(y_{k} \in K_{-}, Q y_{K} \in P^{-1}\left(K_{-}\right) \cap M_{\sim}\right)$. On the other hand $T Q y_{0}=\alpha_{L}(T) Q y_{0}+$ $z_{L}\left(z_{L} \in L_{-}\right)$, and thus $\alpha_{L}(T)=\alpha_{K}(S)$. This proves that the diagonal multiplicity of $\alpha \neq 0$ with respect to $\mathscr{F}(T)$ exceeds that of $\alpha$ with respect to $\mathscr{F}(S)$. This proves the theorem.

3. Superdiagonal forms for compact linear operators. If $T \in L(X)$ is a compact linear operator acting on a complex locally convex space $X$, then (cf. section 1) $T$ is related to a compact linear operator $S \in L(Y)$ acting on a complex Banach space $Y$. J. R. Ringrose [4, cf. proof of Theorem 1] implicitly proved that every maximal invariant nest $\mathscr{F}(S)$ is simple. Hence Theorem 2.1 implies that there exists a simple invariant nest $\mathscr{F}(T)$.

For Banach spaces $X$ the following result is due to Ringrose.

Theorem 3.1 (D. Koros [2, Theorem 2]). Let $T \in L(X)$ be a compact linear operator acting in a complex locally convex space $X$, and let $\mathscr{F}(T)$ be a simple nest of subspaces of $X$, each of which is invariant under $T$. Then of $T$;

(i) a non-zero scalar $\alpha$ is an eigenvalue of $T$ if and only if $\alpha$ is a diagonal coefficient

(ii) the diagonal multiplicity of $\alpha$ is equal to its algebraic multiplicity as an eigenvalue of $T$;

(iii) the operator $T$ is quasi-nilpotent if and only if $\alpha_{M}(T)=0(M \in \mathscr{F}(T))$; or equivalently if and only if $T(M) \subseteq M_{-}(M \in \mathscr{F}(T))$.

Proof. Since $T$ and $S$ are related, $\alpha \neq 0$ is an eigenvalue of $T$ if and only if $\alpha$ is an eigenvalue of $S$ with the same algebraic multiplicity $d(\alpha)$. This is an easy consequence of the definition of related operators (cf. Wrobel [5]). By Theorem 2 of Ringrose [4], $d(\alpha)$ is equal to the diagonal multiplicity of $\alpha$ with respect to $\mathscr{F}(S)$, and hence $d(\alpha)$ is equal to 
the diagonal multiplicity of $\alpha$ with respect to $\mathscr{F}(T)$ by Theorem 2.2. Since $T$ is quasi-nilpotent if and only if $S$ is quasi-nilpotent, (iii) follows from Ringrose's result and Theorem 2.2 as well.

\section{REFERENCES}

1. P. Enflo, On the invariant subspace problem in Banach spaces, Séminaire Maurey-Schwartz (1975-1976). Espaces $L^{\mathfrak{D}}$ Applications Radonifiantes et Géometrie des Espaces de Banach, Exp. No. 14-15, Centre Math., École Polytech., (Palaiseau, 1976).

2. D. Koros, Superdiagonal forms for completely continuous linear operators, Glasgow, Math. J. 23 (1982), 163-170.

3. A. Pietsch, Operator ideals (Berlin, 1978).

4. J. R. Ringrose, Superdiagonal forms for compact linear operators, Proc. London Math. Soc. (3) 12 (1962), 367-384.

5. V. Wrobel, Spektraltheorie beschränkter linearer Operatoren, Math. Nachr. 80 (1977), 151-156.

Mathematisches Seminar

DER UNIVERSITÄT KIEL

Olshausenstrabe 40

D-2300 KIEL 1

Fed. Rep. Germany 